\title{
EFEITO DA TEMPERATURA SOBRE A GERMINAÇÃO DE TRÊS ESPÉCIES DE Pinus CULTIVADAS NO BRASIL ${ }^{1}$
}

\author{
Francisco de Assis Cardoso Almeida ${ }^{2}$, Joaquim Pereira Rodrigues ${ }^{3}$, Silvana Alves de Almeida ${ }^{3}$, Josivanda \\ Palmeira Gomes de Gouveia ${ }^{2}$ e Nilene Rodrigues dos Santos ${ }^{3}$
}

\begin{abstract}
RESUMO - As espécies de Pinus apresentam alto nível de resistência aos incêndios florestais, razão por que são consideradas, por alguns, plantas pirófitas ativas. Daí se perguntar: o comportamento das sementes dessas espécies evoluiu exclusivamente em relação às perturbações produzidas pelo fogo, ou por outras causas que tenham influenciado esse processo, e, ainda, essas espécies desenvolveram respostas adaptativas similares? Para responder a essas perguntas, submeteram-se sementes de três espécies de Pinus a várias temperaturas e tempo de exposição, para simular resposta aos diferentes regimes de calor, avaliando efeitos provocados na germinação das sementes pelas elevadas temperaturas e o efeito das cinzas, tido como elemento mais notável do micro-ambiente em que se desenvolverão, posteriormente, os propágulos. O material vegetal empregado foi adquirido do Instituto de Pesquisas e Estudos Florestais (IPEF) em Piracicaba, SP. Para avaliar a resposta das sementes ao efeito das temperaturas, usou-se um delineamento estatístico inteiramente casualizado com arranjo fatorial. Na ausência de tratamentos térmicos, as sementes apresentaram altas taxas de germinação e forte redução a partir de temperaturas iguais ou superiores a $70^{\circ} \mathrm{C}$, indicando que, realmente, não são plantas pirófitas ativas. Os valores da germinação obtidos com os tratamentos de temperatura igual a $50{ }^{\circ} \mathrm{C}$ diferiram estatisticamente dos da testemunha, e, quando os valores da temperatura foram iguais ou superiores a $110^{\circ} \mathrm{C}$, observou-se importante redução da germinação em todos os tratamentos. A avaliação simultânea do calor e das cinzas evidenciou que existe interação entre os fatores, com inibição total da germinação em alguns tratamentos.
\end{abstract}

Palavras-chave: Intensidade térmica, solução de cinzas e incêndio florestal.

\section{EFFECT OF TEMPERATURE ON GERMINATION OF THREE PinUS SPECIES CULTIVATED IN BRASIL}

\begin{abstract}
The Pinus species present a high level of resistance to forest fires, being considered as active pyrofit plants. For this reason a question is raised: Did the seed behavior of these species develop exclusively due to fire disturbances or by other causes that have influenced this process?, Still, did these species develop similar adaptable answers? To answer these questions, seeds of three species of Pinus species were exposed to different thermal intensities and time of exposition, in order to simulate responses to the different fire regimes, evaluating the effects of high temperatures on the seeds, as well as the effect of ashes, as the most notable element of the micro-environment in which the plants should grow later. The used plant material was acquired from IPEF (Researches and Forest Studies Institute) - Piracicaba, SP. To evaluate seed responses to the temperatures, an entirely randomized statistical design with factorial arrangement was used. In the absence of thermal treatments, the seeds presented high germination rates and strong reduction in temperatures
\end{abstract}

\footnotetext{
${ }^{1}$ Recebido em 05.07.2004 e aceito para publicação em 10.08.2005.

${ }^{2}$ Universidade Federal de Campina Grande/CCT/DEAg. E-mail: <diassis@ deag.ufcg.edu.br>; <josi@ deag.ufcg.edu.br>.

${ }^{3}$ Mestre em Engenharia Agrícola. E-mail: <joaquim@ig.com.br>; <nilene.r.s@yahoo.com.br>.
} 
equal or superior to $70^{\circ} \mathrm{C}$, suggesting that they are not really active pyrofit plants. The germination values obtained with temperature equal to $50{ }^{\circ} \mathrm{C}$ differed statistically from the control, and when the temperature values were equal or superior to $110^{\circ} \mathrm{C}$, an important germination reduction occurred in all treatments. The simultaneous evaluation for heat and ashes showed the existence of an interaction between the factors, with total inhibition of germination in some treatments.

Keywords: Thermal intensity, ash solution, and forest fire.

\section{INTRODUÇÃO}

Vários são os critérios utilizados, nos últimos anos, para se determinarem o momento e a duração de ocorrência de um incêndio florestal. Segundo Carpanezzi (2000), as florestas trazem benefícios diretos ao homem, como madeira, resinas, óleos essenciais, plantas medicinais, frutos e mel; e indiretos, contribuindo para a conservação do solo, o controle dos ventos, a qualidade da vida humana nas cidades, a redução do risco de enchentes e poluição do ar e da água, a polinização nos pomares, o controle biológico de pragas e a manutenção de rios piscosos e, por serem as árvores resistentes ao fogo moderado, de acordo com a sua densidade na floresta (SBS, 2001), são, também, usadas na construção pesada: carpintaria, pavimento, utilidades, linha férrea, barcos, barris, polpa e celulose, entre outros.

A incidência de um fogo coloca as plantas em posição extrema, originando traumas tão profundos que somente lhe sobrevivem os indivíduos que apresentam mecanismos adaptados e impedem sua morte, porém tais mecanismos ocorrem unicamente para os incêndios de baixa intensidade e que avançam rapidamente (VÁSQUEZ e MORENO, 1993).

As plantas, em sua evolução, têm-se adaptado às modificações que incidem com maior ou menor freqüência no meio em que se encontram. Quando esse meio está submetido a mudanças, as plantas buscam a maneira de se reproduzir e multiplicar no tempo e no espaço (KEELEY, 1991). Existe um grupo de plantas denominadas pirófitas ativas (TRABAUD, 1987), em que o fogo ajuda na disposição e propagação, em que se encontram o Cistus ladanifu e alguns Pinus (Pinus pinea). Em muitos casos, o efeito que origina o fogo sobre alguns Pinus promove a abertura das pinhas, liberando as sementes. Tradicionalmente, os Pinus têm sido considerados plantas adaptadas ao fogo, mas o comportamento não se registra a todos eles (BARBERO et al., 1987). Ademais, muitas plantas consideradas pirófitas necessitam de luz para germinar e não apenas do fogo, sendo, assim, consideradas heliófitas pioneiras; nesse sentido, referencia-se que o $P$. pinaster apresenta algumas dificuldades para se restabelecer depois de grandes incêndios (CASTRO et al., 1990). Em análise desses fatos, verificou-se a importância da preservação desse patrimônio, que, em consequiência dos incêndios florestais, são queimados aproximadamente $30.000 \mathrm{~km}^{2}$ de florestas e bosques anualmente só na Amazônia, isto é, quase duas vezes a área desmatada anualmente (DIAS et al., 2002).

Dentre as espécies queimadas, os povoamentos de Pinus têm exibido bom grau de resistência ao fogo (TRABAUD, 1994; NAVEH, 1994). Por essa razão, tais espécies têm sido tradicionalmente consideradas pirófitas ativas (THANOS et al., 1996). De acordo com Carpanezzi (2000), para a recuperação dos ecossistemas degradados se devem aplicar técnicas adequadas a cada local, como espécies, particularmente, e práticas de implantação, manutenção e manejo, e que a maioria das entidades ambientais, públicas ou particulares do Brasil, não está informada ou estruturada para essa finalidade; no entanto, esse tema vem sendo estudado e apoiado por países preocupados com a preservação e a recuperação dos seus ecossistemas. Como exemplo, a Espanha tem investigado a resposta do fogo em diversas espécies arbustivas mediterrâneas, como Rosmarinus officinallis, e diferentes espécies das famílias Cristáceas e Ericácie (GONZÁLEZ-RABANAL e CASAL, 1995), além de numerosas plantas anuais (NEEMÁN e NEEMÁN, 1993), incluindo-se resposta de diversos macromicetos típicos de pinares de $P$. pinaster (FERNÁNDEZ e RODRIGUEZ, 1992). Igualmente, existe trabalho em que se tem estudado a resposta do fogo que algumas pináceas ibéricas, como os $P$. silvestris, P. nigra (HERNANDO, 1997), P. pinea, $P$. pinaster, $P$. halepensis, $P$. canaviensis e $P$. uncinata (GARCIA, 1998), em virtude de crescerem em zonas onde a incidência do fogo é mais elevada.

R. Árvore, Viçosa-MG, v.29, n.5, p.757-765, 2005 
Como já referenciado, os povoamentos de Pinus possuem alto grau de resistência ao fogo, fato atribuído à alta probabilidade de uma pronta recuperação depois de sofrer um incêndio, isto é, acredita-se que, depois de um incêndio, incrementa-se a germinação das sementes de suas árvores. Porém para Thanos et al. (1989) não parece ser dessa forma, ou seja, parece que as sementes de alguns Pinus apresentam problemas de germinação depois de um fogo de elevada intensidade, questionandose sua inclusão no grupo das pirófitas ativas.

Hernando (1997) tem considerado os Pinus mediterrâneos, plantas adaptadas ao fogo e, em boa medida, como elementos oportunistas; no entanto, existe evidência contrária, referenciando que os Pinus espanhóis germinam com menor rapidez sem a ação de choque térmico, perdendo sua viabilidade em certo período de tempo.

Em busca de estratégias de atuação mais adequadas para esses problemas, realizou-se este trabalho, para avaliar o efeito da temperatura e do tempo de exposição na germinação de sementes de Pinus caribaea, $P$. elliotti e P. taeda.

\section{MATERIAL E MÉTODOS}

O presente trabalho foi conduzido no Laboratório de Armazenamento e Processamento de Produtos Agrícolas da Unidade Acadêmica de Engenharia Agrícola do Centro de Ciências e Tecnologia da Universidade Federal de Campina Grande, em Campina Grande, PB.

Utilizaram-se sementes de três espécies de Pinus (P. caribaea, P. elliotti e $P$. taeda) procedentes de regiões que contêm um grupo de árvores de características superiores (porte, produtividade e fruto), produzidas pelo Instituto de Pesquisas e Estudos Florestais (IPEF), Piracicaba, SP, destinada à produção de sementes.

As sementes foram submetidas a diferentes temperaturas $\left(50,70,90,110,130\right.$ e $\left.150^{\circ} \mathrm{C}\right)$ e tempo de exposição (2, 4 e 6 min). Para esta avaliação, quatro amostras de 50 sementes de cada espécie foram levadas à estufa, sobre papel-alumínio; o número de sementes que formavam cada ensaio permaneceu pelo tempo necessário em cada uma das temperaturas. Uma vez transcorrido esse tempo, foram retiradas da estufa e levadas a um dessecador, para equilibrar sua temperatura com a do ambiente. A leitura foi feita diariamente, retirandose as plântulas emergidas com tamanho igual ou superior a $5 \mathrm{~mm}$. Para as espécies de P. elliotti e P. taeda, foi necessário o uso de um fotoperíodo (12 escuro/12 luz) e de estratificação a frio $\left(10^{\circ} \mathrm{C}\right.$ durante 26 dias $)$, após cada tratamento térmico.

Para avaliação do efeito conjunto da intensidade do calor com adição de cinzas, empregou-se cinza obtida pela combustão total em meio aeróbico do material vegetal (folhas, ramos, frutos, caule e cascas) das espécies de Pinus, objeto do estudo.

Os testes de germinação com o emprego de cinzas foram instalados com o mesmo substrato anterior, irrigados com uma solução de cinzas em três concentrações, as quais foram preparadas, pesando-se 15, $30 \mathrm{e} 45 \mathrm{~g}$ de cinzas para cada litro da solução, respectivamente, mediante a pesagem em balança eletrônica. Posteriormente, com a ajuda de um bastão de vidro foram separadas e dissolvidas em $1 \mathrm{~L}$ de água destilada, com a qual se irrigava regularmente o substrato de papel contido em placa de Petri, onde foram semeadas. Nos tratamentos térmicos com o emprego dessas soluções, estas foram previamente submetidas às temperaturas de 25, 70, 90 e $130{ }^{\circ} \mathrm{C}$ e expostas ao tempo de exposição de 2,4 e 6 min, respectivamente.

O teste de germinação foi realizado conforme as Regras para Análise de Sementes (BRASIL, 1992). Nos tratamentos de cinza, o substrato era irrigado com as soluções em diferentes concentrações de cinza. A porcentagem de germinação do Pinus caribaea foi calculada com o total das sementes emergidas no $21^{\circ}$ dia depois da semeadura e a das espécies de $P$. elliotti e P. taeda, no $28^{\circ}$ dia.

O delineamento estatístico empregado nas duas etapas do experimento foi o inteiramente casualizado, disposto em fatorial, sendo o arranjo da primeira etapa $6 \times 3 \times 3$ (seis temperaturas, três tempos de exposição e três variedades); e o da segunda etapa, 4 x $3 \times 3 \times$ 3 (quatro temperaturas, três tempos de exposição, três concentrações de cinza e três variedades), com quatro repetições de 50 sementes, em cada espécie. Os dados foram analisados e as médias, comparadas pelo teste de Tukey a 1 e $5 \%$ de probabilidade. Os dados quantitativos foram submetidos a uma análise de variância na regressão, testando-se polinômios de primeiro e segundo graus para representá-los e considerando a sua significância e o maior valor do coeficiente de correlação.

R. Árvore, Viçosa-MG, v.29, n.5, p.757-765, 2005 


\section{RESULTADOS E DISCUSSÃO}

A análise da variância dos dados da germinação das sementes de Pinus (Tabela 1) apontou valores de F significativos a $1 \%$ de probabilidade, em todos os tratamentos e suas interações, além de efeitos linear e quadrático dos dados quantitativos de todas as espécies estudadas.

Mediante a análise dos dados contidos na Figura 1, tem-se que o comportamento das sementes de Pinus caribaea é completamente diferente dos demais Pinus, visto que a porcentagem de germinação diminuiu a $50{ }^{\circ} \mathrm{C}$, mas aumentou com as temperaturas de 70, 90 e $110^{\circ} \mathrm{C}$, tornando-se nula a partir dos 4 min a 130 ${ }^{\circ} \mathrm{C}$; ademais, houve diferença crescente na germinação, quando passou de 4 para 6 min, como indicado na Figura 1. Segundo Martinéz-Sánchez et al. (1995), uma temperatura em torno dos $70^{\circ} \mathrm{C}$ poderia determinar a não-germinação do $P$. halenpensis se o tempo de exposição fosse superior a $10 \mathrm{~min}$.

Diferentes trabalhos consideraram algumas Pináceas ibéricas termófilas ( $P$. halenpensis e $P$. pinaster), por sua boa regeneração depois de um incêndio florestal (TRABAUD e OUSTRIC, 1989), e comprovou-se que sua germinação não era estimulada pelas temperaturas elevadas, mesmo as que não apresentaram mortalidade significativa (em torno de $250^{\circ} \mathrm{C}$ ), contradizendo a teoria anterior (MARTINÉZ-SÁNCHEZ et al., 1995).

A germinação das sementes dos Pinus estudados diminui com a temperatura, tendo a do Pinus caribaea sido superior à do Pinus elliotti, que suplantou a do Pinus taeda. Na temperatura de $110^{\circ} \mathrm{C}$ e no tempo de exposição de 2 min das sementes a essa temperatura, a germinação do Pinus caribaea $(71 \%)$ foi superior em 24,5\% à dos demais Pinus estudados (germinação média $=54 \%$ ). Observou-se, ainda, uma germinação de 59,7\% das sementes do Pinus caribaea quando expostas a $130^{\circ} \mathrm{C}$ por 2 min e que os demais Pinus não germinaram quando foram submetidos a essas condições (Figura 1).

Na Tabela 2, encontra-se o resumo da análise de variância, na qual se detectou efeito não-significativo de algumas interações, indicando que esses fatores exercem efeito isolado.

R. Árvore, Viçosa-MG, v.29, n.5, p.757-765, 2005
Tabela 1 - Quadrado médio das análises de variância e de regressão da germinação das sementes de três espécies de Pinus

Table 1 -Mean square of the variance and regression analyses of seed germination in threePinus species

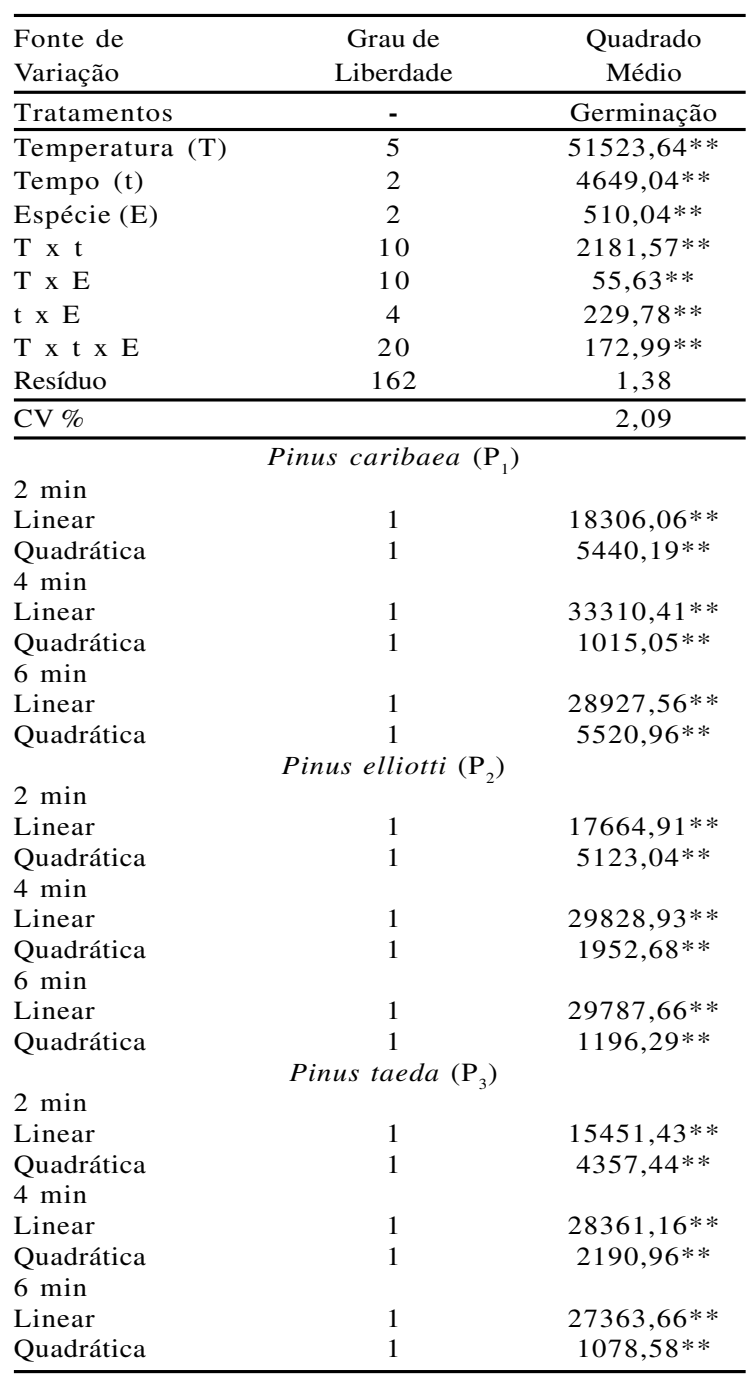

** Significativo a $1 \%$ de probabilidade.

As equações obtidas da análise de regressão na variância revelaram efeito linear nas concentrações de cinza, com todas as espécies, e quadrático em algumas delas. No entanto, para se expressarem os resultados, optou-se pela equação de maior coeficiente de correlação $\left(\mathrm{R}^{2}\right)$, quer de efeito linear, quer de efeito quadrático significativo. 
(a)

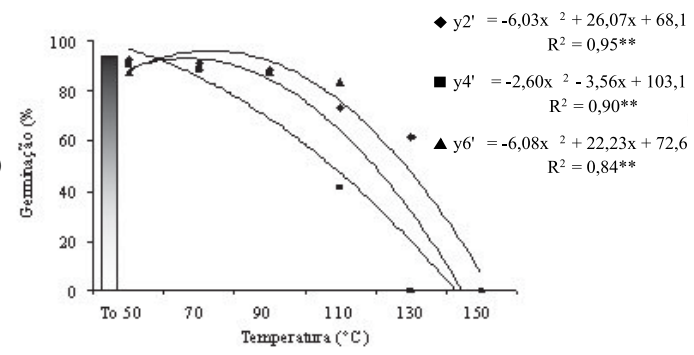

(b)

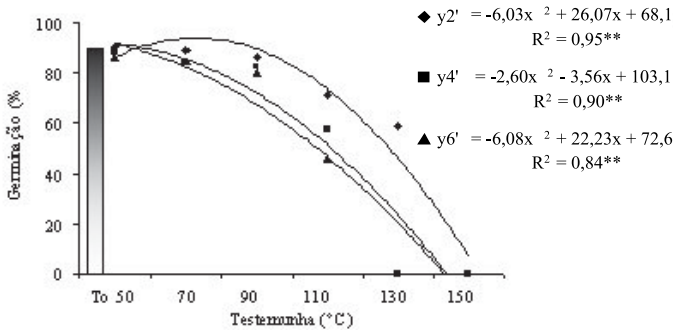

(c)

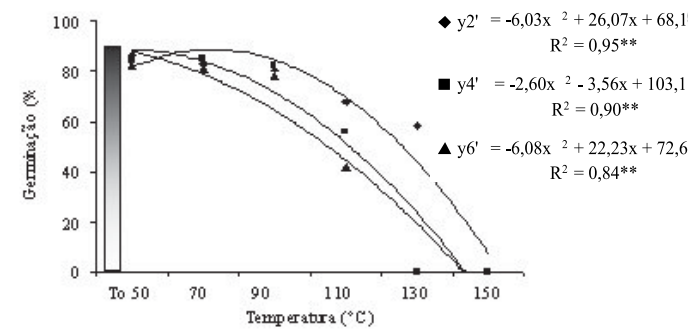

Figura 1 - Efeito da temperatura sobre a germinação das sementes de $P$. caribaea (a), $P$. elliotti (b) e $P$. taeda (c), respectivamente, submetidas a $2,4 \mathrm{e}$ 6 min de exposição a cada temperatura.

Figure 1 - Effect of temperature on seed germination of $P$. caribaea (a), P. elliotti ( $b$ ) and P. taeda (c) exposed for 2, 4 and 6 min to each temperature, respectively.

Com respeito ao efeito induzido pela presença de cinzas na água de irrigação, observou-se perda de germinação devida ao aumento da concentração das cinzas na água de irrigação (Figura 2). No entanto, tem-se igualdade estatística nas concentrações de 30 e $45 \mathrm{~g} \mathrm{~L}^{-1}$, com resultados inferiores de germinação frente à concentração de $15 \mathrm{~g} \mathrm{~L}^{-1}$, na temperatura de $90{ }^{\circ} \mathrm{C}$. Igual comportamento se tem com a intensidade de calor promovido pela temperatura de $70{ }^{\circ} \mathrm{C}$, nas concentrações de 15 e $30 \mathrm{~g} \mathrm{~L}^{-1}$. Com relação ao tempo ( T x t), verificou-se que este não interfere estatisticamente quando as sementes são postas para germinar, à temperatura de $25^{\circ} \mathrm{C}$, entretanto as sementes submetidas a temperaturas de 70 e $90^{\circ} \mathrm{C}$ foram mais afetadas na sua germinação quando expostas, a 6 min, a essas temperaturas. Em estudos realizados com sementes de outras espécies florestais, as cinzas têm causado efeito inibidor ou nulo sobre a germinação (GONZÁLEZRABANAL e CASAL, 1995; REYES e CASAL, 1998), sendo a causa dessa inibição devida à elevação do pH; contudo, Hernando (1997) não encontrou efeito de cinzas sobre a germinação dos $P$. sylvestris e $P$. nigra.

Tabela 2 - Quadrado médio das análises de variância e de regressão da germinação das sementes de Pinus com adição de soluções de cinzas

Table 2 - Mean square of the variance and regression analyses of Pinus seed germinationwith the addition of ash solutions

\begin{tabular}{|c|c|c|c|}
\hline \multirow{2}{*}{$\begin{array}{l}\text { Fonte de Variação } \\
\text { Tratamentos }\end{array}$} & GL & Que & adrado Médio \\
\hline & & & \\
\hline Temperatura (T) & 3 & & $23552,60 * *$ \\
\hline Tempo (t) & 2 & & $4510,89 * *$ \\
\hline Cinzas (c) & 2 & & $6651,84 * *$ \\
\hline Espécie (E) & 2 & & $1089,34 * *$ \\
\hline $\mathrm{T} \times \mathrm{t}$ & 6 & & $1725,21 * *$ \\
\hline $\begin{array}{lll}\mathrm{T} & \mathrm{x} & \mathrm{c} \\
\mathrm{T} & \mathrm{x} & \mathrm{E}\end{array}$ & 6 & & $1105,79 * *$ \\
\hline $\mathrm{T} \times \mathrm{E}$ & 6 & & $131,95 * *$ \\
\hline $\mathrm{t} \times \mathrm{c}$ & 4 & & $16,50 \mathrm{~ns}$ \\
\hline$t \times E$ & 4 & & $63,63 \mathrm{~ns}$ \\
\hline $\mathrm{c} \times \mathrm{E}$ & 4 & & $161,66 * *$ \\
\hline $\mathrm{T} \times \mathrm{t} \times \mathrm{c}$ & 12 & & $149,21 * *$ \\
\hline$\overline{T \times t \times E}$ & 12 & & $41,56 \mathrm{~ns}$ \\
\hline \multirow{2}{*}{$\begin{array}{l}T \times c \times E \\
t \times c \times E\end{array}$} & 12 & & $33,66 \mathrm{~ns}$ \\
\hline & 8 & & $47,53 \mathrm{~ns}$ \\
\hline$T \times t \times c \times E$ & 24 & & $22,58 \mathrm{~ns}$ \\
\hline Resíduo & 324 & & 40,32 \\
\hline \multicolumn{2}{|l|}{$\overline{\mathrm{CV}}(\%)$} & & 13,57 \\
\hline \multirow{2}{*}{\multicolumn{4}{|c|}{$2 \min .15 \mathrm{~g} \mathrm{~L}^{-1}$}} \\
\hline $2 \min .15 \mathrm{~g} \mathrm{~L}^{-1}$ & & & \\
\hline \multicolumn{4}{|l|}{$\begin{array}{l}\text { Quadrática } \\
2 \text { min. } 30 \mathrm{~g} \mathrm{~L}^{-1}\end{array}$} \\
\hline \multicolumn{4}{|l|}{ Linear $\quad 1$} \\
\hline \multicolumn{4}{|l|}{$\begin{array}{l}\text { Quadrática } \\
2 \text { min. } 45 \mathrm{~g} \mathrm{~L}^{-1}\end{array}$} \\
\hline \multicolumn{4}{|l|}{ Linear } \\
\hline \multicolumn{4}{|l|}{$\begin{array}{l}\text { Quadrática } \\
4 \text { min. } 15 \mathrm{~g} \mathrm{~L}^{-1}\end{array}$} \\
\hline \multicolumn{4}{|l|}{ Linear } \\
\hline \\
\hline Linear &, $0 * *$ & $1 * *$ & $4 \mathrm{~min} . \quad 30 \mathrm{~g} \mathrm{~L}^{-1}$ \\
\hline $\begin{array}{l}\text { Quadrática } \\
4 \text { min. } 45 \mathrm{~g} \mathrm{~L}^{-1}\end{array}$ &, $25 * *$ & $5 * *$ & $75625 * *$ \\
\hline Linear & * & ** & 0 , \\
\hline $\begin{array}{l}\text { Quadrática } \\
6 \text { min. } 15 \mathrm{~g} \mathrm{~L}^{-1}\end{array}$ & * & $81,00 \mathrm{~ns}$ & \\
\hline Linear & 16 & & 1436 \\
\hline Quadrática & $5,00 *$ & 25 & 100,0 \\
\hline $6 \mathrm{~min} .30 \mathrm{~g} \mathrm{~L}^{-1}$ & & & \\
\hline Linear & $13364,4 * *$ & $4 * *$ & 11858,4 \\
\hline $\begin{array}{l}\text { Quadrática } \\
6 \text { min. } 45 \mathrm{~g} \mathrm{~L}^{-1}\end{array}$ & $25 \mathrm{mo}$ & $20,25 \mathrm{~ns}$ & \\
\hline Linear & $4,4 * *$ & $0 * *$ & $0 *$ \\
\hline Quadrática & $0,25 \mathrm{~ns}$ & 0,00 & 25,0 \\
\hline
\end{tabular}

**,* Significativos a 1 e $5 \%$ de probabilidade, respectivamente e ${ }^{n s}$ não-significativo. 
(a)
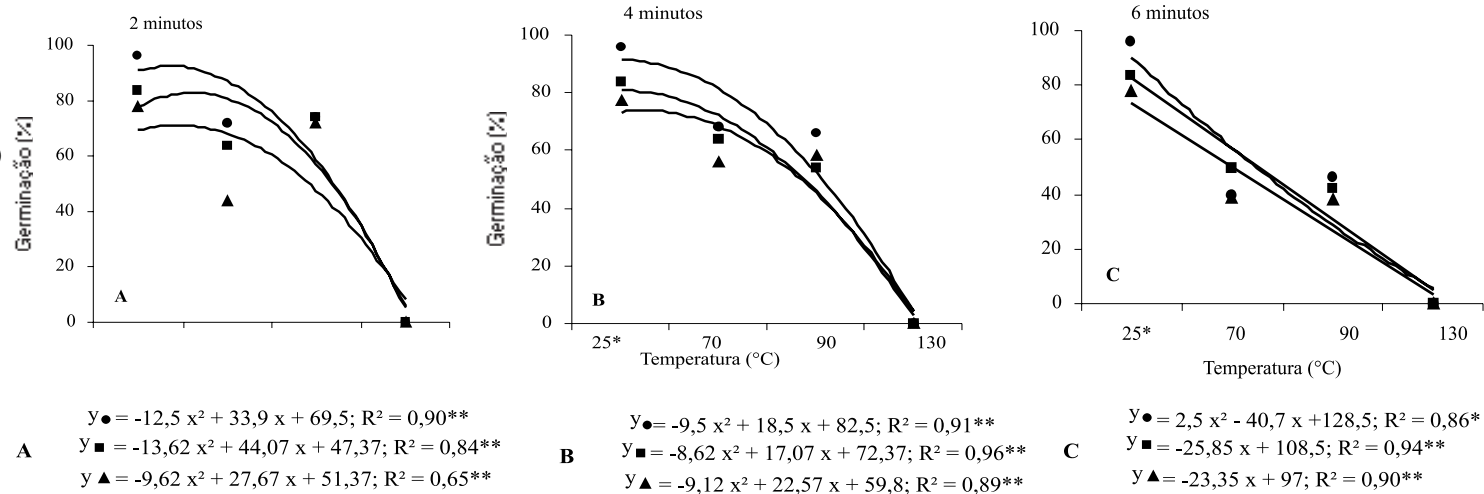

(b)
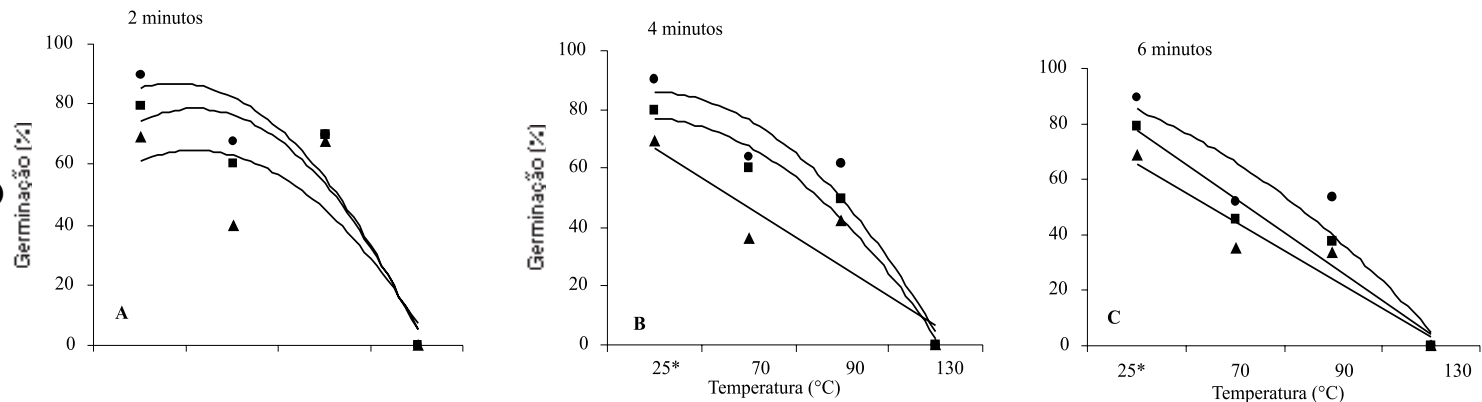

$\begin{aligned} & \mathrm{y}_{\bullet}=-12 \mathrm{x}^{2}+33,2 \mathrm{x}+64 ; \mathrm{R}^{2}=0,90 * * \\ & \text { A } \mathrm{y}_{\mathbf{}}=-12,62 \mathrm{x}^{2}+40,27 \mathrm{x}+46,37 ; \mathrm{R}^{2}=0,84 * * \\ & \mathrm{y}_{\mathbf{\Delta}}=-9,75 \mathrm{x}^{2}+30,85 \mathrm{x}+40,25 ; \mathrm{R}^{2}=0,62 * *\end{aligned}$

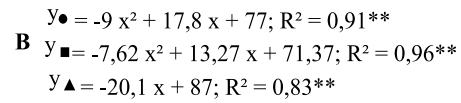

$\mathrm{y} \bullet=-4 \mathrm{x}^{2}-6,8 \mathrm{x}+96 ; \mathrm{R}^{2}=0,88^{* *}$

C $\mathrm{y}=-24,65 \mathrm{x}+102,5 ; \mathrm{R}^{2}=0,95 * *$

y $\Lambda=-20,8 \mathrm{x}+86,5 ; \mathrm{R}^{2}=0,90^{* *}$

(c)
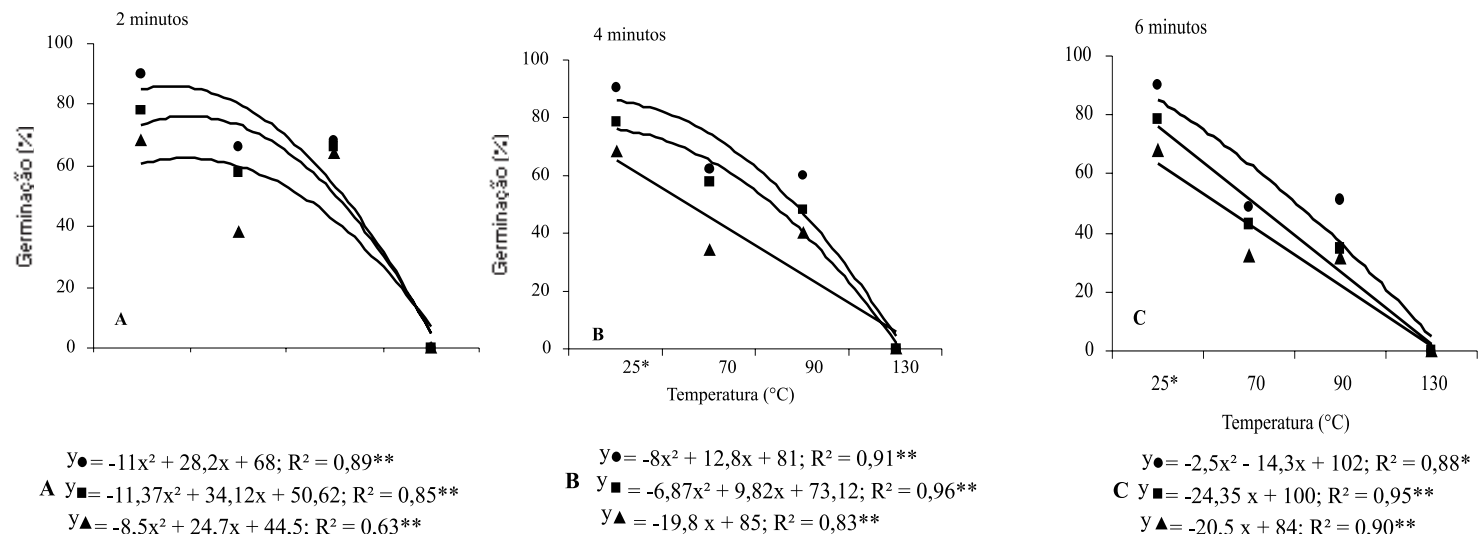
$\mathrm{y} \bullet=-8 \mathrm{x}^{2}+12,8 \mathrm{x}+81 ; \mathrm{R}^{2}=0,91 * *$
B $\mathrm{y}_{\mathbf{m}}=-6,87 \mathrm{x}^{2}+9,82 \mathrm{x}+73,12 ; \mathrm{R}^{2}=0,96 * *$
$\mathrm{y}_{\boldsymbol{\Lambda}}=-19,8 \mathrm{x}+85 ; \mathrm{R}^{2}=0,83^{* *}$

$\mathrm{y}_{\bullet}=-2,5 \mathrm{x}^{2}-14,3 \mathrm{x}+102 ; \mathrm{R}^{2}=0,88^{*}$

C $\mathrm{y}=-24,35 \mathrm{x}+100 ; \mathrm{R}^{2}=0,95^{* *}$

$\mathrm{y} \boldsymbol{\Delta}=-20,5 \mathrm{x}+84 ; \mathrm{R}^{2}=0,90 * *$

Figura 2 - Germinação de P. caribaea (a), P. elliotti (b) e P. taeda (c) em temperaturas, concentrações de cinzas $(\bullet 15$, 30, $\left.\Delta 45 \mathrm{~g} \mathrm{~L}^{-1}\right)$ e tempo de exposição.

Figure 2-Germination of P. caribaea (a), P. elliotti (b) and P. taeda (c) in different temperatures, ash concentration ( 15 , $\left.\boldsymbol{\square} 30, \boldsymbol{\Delta} 45 \mathrm{~g} \mathrm{~L}^{-1}\right)$ and time of exposition.

R. Árvore, Viçosa-MG, v.29, n.5, p.757-765, 2005 
Para Reyes e Boedo (1999), o efeito das cinzas sobre a germinação é diferente em cada espécie. Levando em consideração esses comentários, considera-se que os Pinus estudados apresentam boa seleção ecológica e biológica para colonizar áreas depois de diferentes alterações, mas não depois de incêndios intensos, especialmente em se tratando de incêndios de copa. Sobre esse tema, Keeley e Zedler (1978) afirmaram que as plantas, em sua seleção, têm-se adaptado às alterações que incidem, com maior ou menor freqüência, no meio em que se desenvolvem. Nesse meio, quando submetido periodicamente a diferentes alterações, as plantas procuram a maneira de se reproduzirem e multiplicar no tempo e no espaço, mediante o fenômeno da dormência.

Quando os incêndios afetam uma mesma área em intervalos regulares de tempo longo maiores que 100 anos, as plantas se adaptam, de forma que basicamente se reproduzem a partir de sementes. Porém, se o intervalo de retorno é menor que 25 anos, as plantas que habitam essa região se reproduzem, principalmente, por rebrotas (CHRISTENSEN, 1985). Ao mesmo tempo, as plantas podem alcançar diferentes níveis de adaptação quanto à sua regeneração, segundo a forma de propagação do calor, favorecendo sua regeneração, de maneira que existem plantas indiferentes a esse efeito; sua adaptação é passiva e consiste em dificultar o início dos incêndios, como é o caso das possuidoras de cascas grossas, elevada concentração de sais e baixa inflamabilidade, entre outras plantas que possuem rizomas ou tubérculos, cuja proteção é estimulada pela ação do calor. Em muitos casos, o efeito que produz o fogo é o que faz abrir suas pinhas resinosas, propagando as sementes. Assim, Trabaud (1987) introduziu o termo "adaptado ao fogo" como mais correto para descrever esse comportamento.

Tem-se observado que a germinação dos Pinus é estimulada pela luz através do sistema de fotocromos (THANOS e SKORDILIS, 1987), comprovado pela elevada densidade de indivíduos que aparecem nas margens ao abrir uma pista florestal, atravessando a área plantada com os Pinus (TOOLE, 1973). Por isso, a eliminação da cobertura vegetal durante um incêndio poderia favorecer o desenvolvimento de espécies heliófilas, como as estudadas por Trabaud et al. (1985), e explicaria que, durante o incêndio, as temperaturas suportadas pela maioria das sementes alcançam valores superiores a $90^{\circ} \mathrm{C}$, o que, como comprovado, é letal para os embriões dos Pinus.

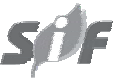

\section{CONCLUSÕES}

Mediante os resultados do presente trabalho, conclui-se que:

a) Na temperatura de $130^{\circ} \mathrm{C}$ e tempo de exposição de 6 min, a germinação dos Pinus foi uniforme ( $\mathrm{M}=$ $19,9 \%)$.

b) A germinação média das sementes diminuiu com o aumento da intensidade do calor e o tempo de exposição.

c) Os polinômios representaram satisfatoriamente os dados experimentais da germinação dos Pinus diante da intensidade de calor e do tempo de exposição.

d) A germinação das sementes de Pinus não foi afetada quando estas foram submetidas a temperaturas menores e iguais a $50{ }^{\circ} \mathrm{C}$.

e) O comportamento das sementes de Pinus caribaea é completamente diferente dos demais Pinus estudados ( $P$. elliotti e $P$. taeada); sua germinação diminui a $50^{\circ} \mathrm{C}$, mas aumenta quando os choques ocorrem a temperaturas de 70,90 e $110^{\circ} \mathrm{C}$, aos 6 min de exposição, tornando-se nula a partir dos $4 \mathrm{~min}$ a $130{ }^{\circ} \mathrm{C}$.

f) Os tratamentos térmicos aplicados em combinação com as concentrações de cinza apresentaram redução na germinação de todas as espécies, inibindo-as completamente em tratamentos com adição de concentrações de cinzas maiores que $30 \mathrm{~g} \mathrm{~L}^{-1}$.

g) Os resultados indicam que o fogo não pode ser um agente potenciador na germinação dos Pinus estudados e as espécies não são plantas pirófitas.

\section{REFERÊNCIAS BIBLIOGRÁFICAS}

BARBERO, M. et al. Incidence of exogenous factors on the regeneration of Pinus halepensis after fires. Ecologia Mediterrânea, v.13, p.40-51, 1987.

BRASIL. Ministério da Agricultura. Regras para análise de sementes. Brasília: SNDA/ DNDV/CLAV, 1992.365p.

CARPANEZZI, A.A. Benefícios imediatos da floresta. In: SEMINÁRIO SOBRE ATUALIDADES EM PROTEÇÃO FLORESTAL, 2000, Curitiba. Anais... Curitiba: 2000.

R. Árvore, Viçosa-MG, v.29, n.5, p.757-765, 2005 
CASTRO, J.F.; BENTO, J.; REGO, F. Regeneration of Pinus pinaster Forest after wildfires. In: GOLDAMMER, J.G.; JENKINS, M.J. (Eds.). Fire in ecosystem dynamics, The Hague: SPB Academic Publishing, 1990. p.71-75.

CHRISTENSEN, N.L. Shrubland fire regimes and their evolutionary consequences. In: PICKETTES, S.T.A.; WHITE, P.S. (Eds.). The ecology of natural disturbance and patch dynamics. New York: Academic Press, 1985. p.85-100.

DIAS, M.C.V. et al. Relatório do Instituto de Pesquisa Ambiental (IPAM) em colaboração com o Instituto de Pesquisa Econômica Aplicada (IPEA) e o Centro de Pesquisa Woods Hole (WHRC), 2002.

FERNÁNDEZ, A.M.F.J.; RODRIGUEZ, A. El fuego y la respuesta de los macromicetos del suelo en pinares de Pinus pinaster, Ait

Investigaciones Agrarias Sistema de Recourse Forestale, v.1, n.2, p.137-150. 1992.

GARCIA, M.V.S. Probabilidad de germinación después de choques térmicos en los representantes Españoles del género Pinus. Madri: 1998. 105p.

GONZÁLEZ-RABANAL, F.; CASAL, M. Effect of high temperatures and ash on germination of ten species from gorse shrubland. Vegetatio, v.116, p.123-131,1995.

HERNANDO, B.S. Efecto del fuego en la germinación de Pinus sylvestris L. y de Pinus nigra Arn. Subsp. Salzmannii (Dunal). Madri: 1997. 81p.

KEELEY, J.E. Seed germination and life history syndromes in the California chaparral. The Botanical Review, v.57, p.81-116, 1991.

KEELEY, J.E.; ZEDLER, P.H. Reproduction of chaparral shrubs after fire: a comparison of sprouting and seeding strategies. American Midland Naturalist, n.99, p.142-161, 1978.

MARTÍNEZ-SÁNCHEZ, J.J. et al. Effects of high temperatures on germination of Pinus halepensis mill. And Pinus pinaster seeds in southest Spain. Vegetatio, v.116, p.69-72, 1995.

R. Árvore, Viçosa-MG, v.29, n.5, p.757-765, 2005
NAVEH, Z. The role of fire and its management in conservation of Mediterranean ecosystem and landscapes. In: MORENO, J.M.; OECHEL, W.C. (Eds). The Role of fire in mediterranean-type ecosystems. Berlin: Springer Verlag, 1994. p.163-186.

NEEMÁN, M.; NEEMÁN, G. The effect of ash on the germination and early growth of shoots and roots of Pinus, Cistus and annuals. Seed Science and Technology, v.21, p.339-349, 1993.

REYES, O.; BOEDO, M. El fuego como controlador de la germinación de Cytisus striatus y de $C$. Multiflorus y su aplicación agronómica, Santiago de Compostela. 1999. Disponível: http:// www.juntadeandalucia.es/medioambiente/ ponencias/24.htm. Acesso em: 30/04/2003.

REYES, O.; CASAL, M. Germination of Pinus pinaster, $P$. radiata and Eucalyptus globuues in relation to the amount of ash produced in forest fires. Sciences Florestiéres, n.55, p.837-845, 1998.

SOCIEDADE BRASILEIRA DE SILVICULTURASBS. Participação do Brasil no mercado internacional. 2001. Disponível: http://www.sbs.org.br/estatisticas.html. Acesso em: 30/04/2003.

THANOS, C.A.; DASKALAKOU, E.N.; NIKOLAIDOU, S. Early post fire regeneration of a Pinus halepensis forest at Mt. Parnes, Attica, Greece, Journal Vegetal Science, n.7, p.273280, 1996.

THANOS, C.A.; MARCOU, S.; CHRITODOULAKIS, D.; YANNITSAROS, A. Early post-fire regeneration in Pinus brutia forest ecosystems of Samos island (Greece). Acta Oecology Plant, v.10, p.79-94, 1989.

THANOS, C.A.; SKORDILIS, A. The effects of light, temperature and osmotic stress on the germination of Pinus halenpensis and P. brutia seeds. Seed Science and Technology, v. 15, p.163-174, 1987. 
TOOLE, U.K. Effects of light temperature and their interation on the germination of seeds. Seed Science and Technology, v.1, p.393396, 1973.

TRABAUD, L. Fire and survival traits in plants. In: TRABAUD, L. (Ed.). The role of fire in ecological systems. The Hague: Academic Publishers, 1987. p.65-91.

TRABAUD, L. Post-fire community dynamics in the Mediterranean basin. In: MORENO, J.M.; OCECHEL, W.C. (Eds.). The role of fire in Mediterranean-Type Ecosystems. Berlin: Springer-Verlag, 1994. p.1-15.
TRABAUD, L.; MICHELS, C.; CROSMAN, J. Recovery of burnt Pinus halenpensis Mill. Forests II. Pine reconstitutionafter wildfire. Forest Ecology Manageman, v.13, p.167-179, 1985.

TRABAUD, L.; OUSTRIC, J. Influence du feu sur la germination de semences de quatre especes ligneuses mediterranes a reproduction sexuee oblegatoire. Seed Science and

Technology, v.17, p.589-599, 1989.

VÁZQUEZ, A.; MORENO, J.M. Sensitivity of fire occurrence to Meteorologycal Variables in Mediterranean and Atlantic areas of Spain. Landscape and Urban Planning, v.24, p.129-142, 1993. 